\title{
Broadband extended source imaging Mueller-matrix polarimeter
}

\author{
Juan Manuel lópez-Téllez, ${ }^{1,}{ }^{*}$ Russell A. Chipman, ${ }^{1}$ Lisa W. LI, ${ }^{1}$ Scott C. \\ MCEldowney, ${ }^{2}$ AND MATtheW H. SMIth ${ }^{3}$ \\ ${ }^{1}$ College of Optical Sciences, University of Arizona, 1630 E. University Blvd., Tucson, Arizona 85721, USA. \\ ${ }^{2}$ Facebook Reality Labs. \\ ${ }^{3}$ Axometrics, Inc., 103 Quality Circle, Suite 215, Huntsville, Alabama 35806, USA. \\ *Corresponding author: jm.lopeztellez@gmail.com
}

Received XX Month XXXX; revised XX Month, XXXX; accepted XX Month XXXX; posted XX Month XXXX (Doc. ID XXXXX); published XX Month XXXX

\begin{abstract}
An imaging Mueller-matrix polarimeter, named the RGB950, takes images of medium size (tens of $\mathrm{cm}$ ) objects by using a very bright source, large polarization state generator, and very high quality camera. Its broadband extended light source switches between red, green, blue, and near-infrared light to allow taking polarimetric images for comparison with RGB camera images. The large diffuse source makes shadow transitions gradual and spreads out the specular reflected spot into a larger less conspicuous area. (C) 2019 Optical Society of America
\end{abstract}

OCIS codes: (120.5410) Polarimetry; (260.5430) Polarization; (100.2960) Image analysis; (110.5405) Polarimetric imaging.

http://dx.doi.org/10.1364/OL.99.099999

Polarimetry has a wide variety of scientific and technological applications including ellipsometry [1], polarization element testing [2, 3], machine vision [4], remote sensing [5], industrial control [6], and medical [7] applications. Polarimetric imaging can improve analytic techniques such as image segmentation $[8,9]$ and object recognition in manmade or natural environments $[10,11]$ since it can reveal contrast that does not appear in classical intensity imaging. Polarization information is different from color information since polarization in a scattering sample arises from the direction of electron oscillation while color arises from the quantum energy levels [12]. Thus the information content and contrast mechanisms are different between intensity and polarization [13]. Active Mueller-matrix polarimetric imaging systems illuminate the scene with several controlled polarization states and analyze the polarization state of the light scattered by the scene. At least 16 polarization measurements are required to calculate a complete Mueller-matrix image, which contains the diattenuation, retardance, polarizance, and depolarization information at each pixel [14]. Many Mueller matrix imaging systems having dynamic capabilities in wavelength, angle of incidence, and angle of scatter have been reported [15-17]. In this paper, a design for an imaging Mueller- matrix polarimeter with a broadband extended light source is described and its first results are shown.

Typically, the extent of Mueller-matrix polarimeter images is limited by the size of the polarization optics and source brightness. Monochromator sources are weak and can only illuminate small areas on the order of centimeters. Laser sources are brighter, but speckle becomes an acute issue limiting accuracy since polarimeters need to accurately subtract images during processing. Therefore, in many cases, generating a Mueller Matrix image of a region larger than $1 \mathrm{~cm} x$ $1 \mathrm{~cm}$ requires image stitching and/or repositioning of samples. The literature describes studies of small structures and miniature models of real-world objects applying polarimetry to analysis of surface orientation [18], material [19], and general shapes-features of larger objects [20,21]. Few polarization studies have been conducted on fullsized objects and scenes [10, 19, 21].

The RGB950 Imaging Mueller-matrix Polarimeter described here incorporates a 10 centimeter diameter polarization generator and a large LED array operating at 441, 524, 662, and $947 \mathrm{~nm}$. These wavelengths align with the typical RGB camera spectral response. The camera optics in the polarization state analyzer has an angular field of view of 49.2 degrees (diagonal). These features enable the user to measure typical household, office, and laboratory objects in their full size. The RGB950 is being applied to the studies of complex shapes/object compositions, high surface curvature, and non-uniform illumination.

One advantage of the large source and aperture is shadow reduction. Collimated light beams produce sharp shadows and small specular reflections. In contrast, the large diverging beam from our custom light-box produces gradual shadows and more diffuse specular beams. Furthermore, by using a non-coherent light source, speckle issues for light scattering studies are avoided.

The RGB950 Polarimeter was constructed for the University of Arizona by Axometrics Inc. (Huntsville AL) and is currently operating in the Polarization Laboratory at the University's College of Optical Sciences. This instrument is a modified version of the AxoStep Imaging Mueller Matrix Polarimeter [22]. The RGB950 polarimeter consists of four modules: the LED light-box, a Polarization State Generator (PSG), sample for Mueller matrix analysis, and a Polarization State Analyzer 
(PSA) composed, principally, by a rotating waveplate and a linear polarizer as shown in Figure 1. Waveplates and polarizers are custommade devices. The waveplates are custom multi-plate achromatic retarders whose retardance is approximately 120 degrees across a 400-1000 nm wavelength range, which is consistent with the optimum value shown in Reference [23]. The PSG determines the polarization state of the incident light, whereas the PSA measures the change in this state after interaction with the sample. A PixelLink PLB741G camera used as a photodetector measures the intensity of the light transmitted by the optical system. In transmission the RGB950 can measure samples up to $10 \mathrm{~cm}$ in size, but this instrument is principally applied to studies of scattering from samples.

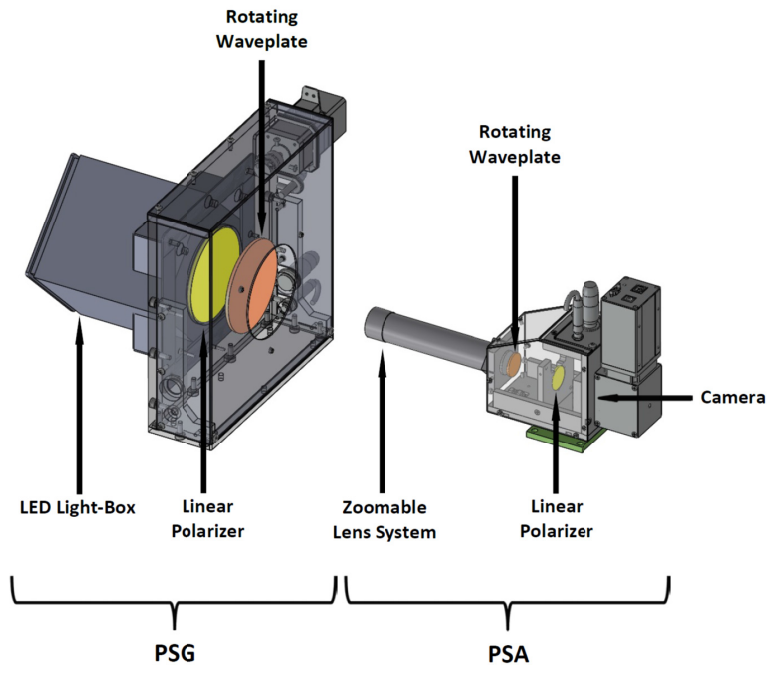

Fig. 1. RGB950 Imaging Mueller-matrix polarimeter setup.

The polarimeter configuration is a dual rotating retarder polarimeter [24]. A sequence of intensity images are acquired as retarders rotate within the PSG and the PSA heads. These images are then processed to calculate the 16 two-dimensional arrays that comprise the Muellermatrix image of the sample which characterizes all of the polarization transformations for that sample. The measurement time depends on two parameters: the exposure time of the camera and the number of measurements for each shot. The exposure time can be set from $0.04 \mathrm{~ms}$ to $400 \mathrm{~ms}$. The minimum measurement time for each Mueller matrix image is approximately 20 seconds and the maximum measurement time is over 3 minutes.

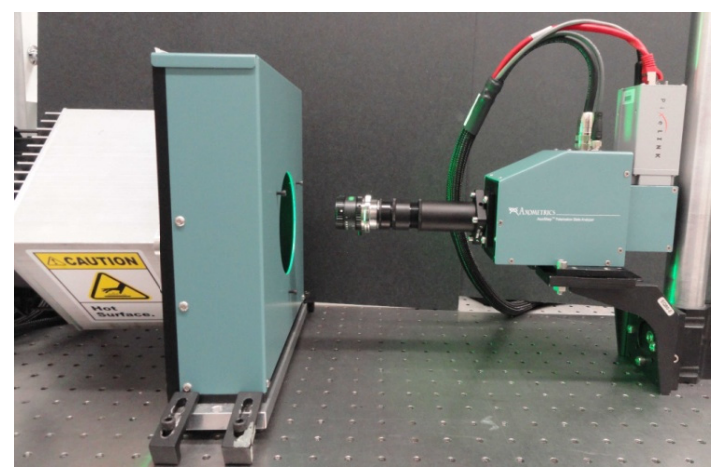

Fig. 2. Photograph of the Polarization State Generator (left) and Polarization State Analyzer (right).
The PSG (Figures 1 and 2) has a large polarizer and large rotating retarder (10 centimeters) to illuminate a large area with a significant amount of light. The light-box produces an expansive light beam. Table 1 shows the spot size as a function of the distance from the PSG aperture. The spot size is defined as the diameter of the light beam itself and it was measured using a ruler and a white screen.

Table 1. Spot size as a function of the distance from the RGB950 PSG aperture.

\begin{tabular}{cc}
$\begin{array}{c}\text { Distance from the } \\
\text { PSG aperture }(\mathbf{c m})\end{array}$ & $\begin{array}{c}\text { Spot size } \\
\text { (cm) }\end{array}$ \\
\hline 0 & 10 \\
5.5 & 20 \\
8 & 29 \\
10.5 & 37 \\
13 & 44 \\
15.5 & 50 \\
18 & 58 \\
20.5 & 64 \\
23 & 72 \\
\hline
\end{tabular}

Four banks of LEDS, blue, green, red, and NIR are switched on one band at a time. The RGB950 light-box spectrum is shown in Table 2, and Table 3 shows the irradiance of the light box at 5,26, and 127 centimeters from the PSG aperture. Even at one meter, it is nearly blinding when looking straight into the polarization generator. When measuring faces, opaque eye cups are typically worn. This generates more than adequate light for measuring scattering from $30 \mathrm{~cm}$ scale objects.

Table 2. RGB950 light-box spectrum.

\begin{tabular}{ccc}
\hline Color & Wavelength (nm) & FWHM (nm) \\
\hline Red & 662.376 & 22.34 \\
Green & 523.92 & 34.63 \\
Blue & 451.095 & 19.65 \\
Infra-red & 947.155 & 39.7 \\
\hline
\end{tabular}

The available pixel resolutions for the images are: 40x32, 80x64, 160x128, 320x256, and 640x512. The RGB950 PSA includes a zoomable lens system (Computar M0814-MP2 2/3" 8 mm f1.4) with adjustable aperture and focusing system (Figure 1).

Table 3. RGB950 light-box irradiance at 5, 26, and 127 centimeters from the PSG aperture.

\begin{tabular}{|c|c|c|c|}
\hline $\begin{array}{l}\text { Wavelength } \\
\text { (nm) }\end{array}$ & $\begin{array}{c}\text { Irradiance } \\
\text { at } 5 \mathrm{~cm} \\
\left(\mathrm{~W} / \mathrm{m}^{2}\right)\end{array}$ & $\begin{array}{c}\text { Irradiance } \\
\text { at } 26 \mathrm{~cm} \\
\left(\mathrm{~mW} / \mathrm{m}^{2}\right)\end{array}$ & $\begin{array}{l}\text { Irradiance } \\
\text { at } 127 \mathrm{~cm} \\
\left(\mathrm{~mW} / \mathrm{m}^{2}\right)\end{array}$ \\
\hline 451.095 & 11 & 347.05 & 131.06 \\
\hline 523.92 & 3.44 & 119.92 & 35.27 \\
\hline 662.376 & 22.15 & 688.47 & 235.60 \\
\hline 947.155 & 23.98 & 761.83 & 275.10 \\
\hline
\end{tabular}

Mueller matrix polarimeter calibration and accuracy are often obtained with measurements in transmission mode for a gap of air and known samples such as a linear polarizer and waveplates. For the RGB950 we use an element-modeling equation of a measurement made in air as the calibration, and we compensate systematic errors using Mueller-matrix baseline image in air. Equations 1 to 4 show the mean value and standard deviation [25] of each Mueller-matrix component over all pixels of the image of air at 451, 524, 662, and 947 nm; respectively. Each Mueller matrix was measured from a 320 x 256 pixel image taken by using the experimental setup shown in Figure 2. A 

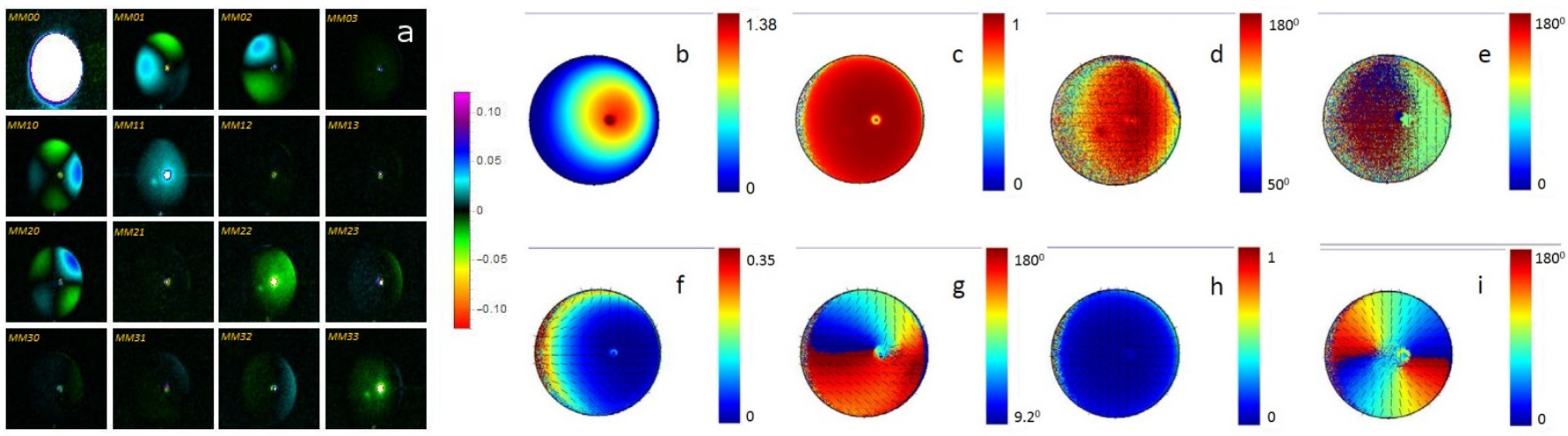

Fig. 4. Mueller matrix (a) and polarimetric properties of a billiard pool ball using light with a wavelength of 662 nm: reflectance in arbitrary units (b), depolarization index (c), total retardance (d), retardance orientation (e), total diattenuation (f), diattenuation orientation (g), total polarizance (h), and polarizance orientation (i). The lines pattern on images (d-i) represents the orientation of that polarization property. The image of the Mueller matrix first element, MM00, is out of scale due to higher intensity values than the other elements. The Mueller-matrix values are not normalized with respect to the MM00 element to show a detailed image of the other elements. The angle between illumination axis and camera axis is 30 degrees.

set of 10 successive measurements was averaged for each wavelength. As a result, all the four Mueller matrices are close to the identity. The maximum deviation from the identity matrix is less than $2 \%$. For comparison, the Mueller-matrix values were normalized with respect to the MM00 element.

Air $M M_{451 \mathrm{~nm}}$

$=\left(\begin{array}{c}1 \pm 0.014 \\ -0.003 \pm 0.002 \\ -0.002 \pm 0.001 \\ 0 \pm 0.003\end{array}\right.$

$-0.001 \pm 0.001$

$0.997 \pm 0.014$

$0.015 \pm 0.002$

$0.005 \pm 0.002$

$$
\begin{gathered}
-0.002 \pm 0.001 \\
-0.013 \pm 0.003 \\
1 \pm 0.014 \\
0.009 \pm 0.002
\end{gathered}
$$
$-0.001 \pm 0.001$
$-0.001 \pm 0.004$
$0.01 \pm 0.004$
$1 \pm 0.014$

(1)

$$
\begin{aligned}
& \text { Air } M M_{524 n m} \\
& =\left(\begin{array}{c}
1 \pm 0.015 \\
0.004 \pm 0.002 \\
0 \pm 0.002 \\
-0.002 \pm 0.005
\end{array}\right.
\end{aligned}
$$$$
-0.001 \pm 0.001
$$$$
0.997 \pm 0.016
$$$$
0.006 \pm 0.004
$$

$0.007 \pm 0.005$

$$
\begin{gathered}
-0.002 \pm 0.001 \\
-0.014 \pm 0.004 \\
0.996 \pm 0.014
\end{gathered}
$$

$-0.004 \pm 0.002$
$-0.002 \pm 0.001$
$-0.007 \pm 0.009$
$0.005 \pm 0.006$

$0.993 \pm 0.014$

(2)

$$
\begin{aligned}
& \text { Air } M M_{662 n m} \\
& =\left(\begin{array}{cccc}
1 \pm 0.021 & 0 \pm 0.001 & -0.001 \pm 0.001 & 0 \pm 0.001 \\
0.002 \pm 0.001 & 0.999 \pm 0.022 & -0.015 \pm 0.003 & -0.011 \pm 0.008 \\
0.002 \pm 0.001 & 0.007 \pm 0.001 & 0.997 \pm 0.02 & -0.001 \pm 0.004 \\
-0.002 \pm 0.004 & 0.005 \pm 0.003 & -0.001 \pm 0.004 & 0.993 \pm 0.022
\end{array}\right)
\end{aligned}
$$

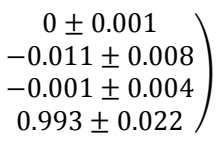

(3)

Air $M M_{947 \mathrm{~nm}}$

$=\left(\begin{array}{c}1 \pm 0.023 \\ 0.002 \pm 0.004 \\ 0.001 \pm 0.005 \\ -0.002 \pm 0.004\end{array}\right.$

$0.002 \pm 0.004$

$0.993 \pm 0.023$

$0.011 \pm 0.007$

$0.001 \pm 0.007$

$0.003 \pm 0.004$
$-0.016 \pm 0.005$
$0.986 \pm 0.02$
$0.003 \pm 0.012$

$-0.001 \pm 0.002$

$-0.004 \pm 0.013$

$0 \pm 0.007$

$0.989 \pm 0.026$

(4)

One scatter polarimetric study was performed on a set of spheres and near spheres of different size, color and material. The goal is to relate the polarimetric properties of the samples with its specific geometry by examining reflectance, depolarization index, diattenuation magnitude and orientation, polarizance magnitude and orientation, and the retardance components. The polarimetric properties images were calculated using Axometrics software along with additional Mathematica scripts developed at the University of Arizona. The polarimetric properties of the measured samples were calculated using the Lu-Chipman polar decomposition [26]. Results for light scattered from a billiard pool ball illuminated at $662 \mathrm{~nm}$ at 30 degrees from the camera axis (Figure 3) are shown using the Mathematica scripts in Figure 4(a), whereas the images shown in Figures 4(b-i) were obtained using Axometrics software. A set of animations for the polarimetric properties of a rotating ceramic jug (Figure 5) can be found in Visualization 1 (Mueller matrix), Visualization 2 (reflectance), Visualization 3 (depolarization index), Visualization 4 (total retardance), Visualization 5 (retardance orientation), Visualization 6 (total diattenuation), Visualization 7 (diattenuation orientation), Visualization 8 (total polarizance), and Visualization 9 (polarizance orientation); these animations were created using Mathematica. The Mueller-matrix image of the billiard ball, Fig. 4(a), shows patterns with black bands in some elements on the first row and first column due to the effect of the Fresnel equations [12]. The polarimetric properties of the billiard ball and the ceramic jug show a depolarization index value close to 1, which correspond to a non-depolarizing optical media [27]. Moreover, the polarizance value is close to 0 , which means that the system under study does not have the ability to polarize unpolarized incident light [26]. The total retardance value for these objects is close

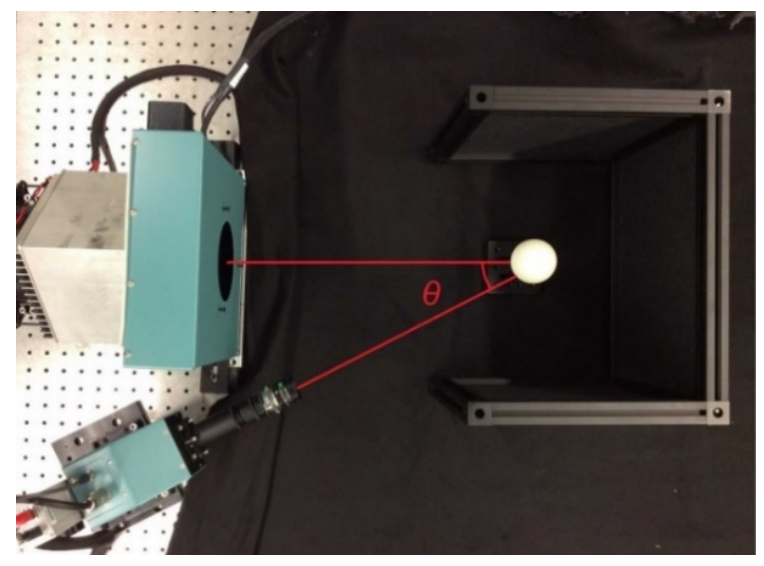

Fig. 3. Experimental setup for measuring a billiard pool ball in scattering mode. $\theta$ represents the angle between illumination axis and camera axis. The sample is a white ball with $5.71 \mathrm{~cm}$ in diameter. Blackout materials such as a black fabric and a black back ground screen were used to isolate the sample under study from undesirable or spurious effects due to the surrounding reflecting/scattering conditions. 
to 180 degrees as typical of scattering objects. Additionally, the polarization orientation shows patterns that can be related to the geometry of the samples [28]. A more complete analysis and discussion about the results of this polarimetric study will be shown in a future publication.

In conclusion, a custom Imaging Mueller-matrix Polarimeter is operating as a facility for measuring the light scattered from medium size (tens of $\mathrm{cm}$ ) objects with a broadband bright source, large polarization state generator, and a very high quality camera. In contrast to instruments with collimated light beams, the large diverging beam from the RGB950 light-box produces gradual shadows and diffuse specular beams. Moreover, by using a non-coherent light source, speckle issues in light scattering studies are avoided. The accuracy of the measurements and high quality images taken by this instrument permit taking Mueller-matrix images of human faces, furniture, and other large objects. This polarimeter addresses polarimetric challenges in the analysis of complex shapes/object compositions with high surface curvature and non-uniform illumination by reducing shadowing and enlarging and blurring the specularly reflected spot. To achieve these characteristics, the light propagates at significant angles (up to 20 degrees) through the PSG and PSA elements and the highly diverging beam generates substantial ambient light from nearby objects. These design choices are different from the choices made for polarimeters to measure retarders, polarizers, liquid crystals, optical elements, or ellipsometric samples; measurements which can require polarimetric accuracy better than $0.1 \%$. In contrast, the RGB950 is typically measuring scattering from paint, wood, plastic, metal, skin, and similar objects which display considerable inhomogeneity variation from point to point or sample to sample. Thus the goal of RGB950 measurements is typically $0.5 \%$.

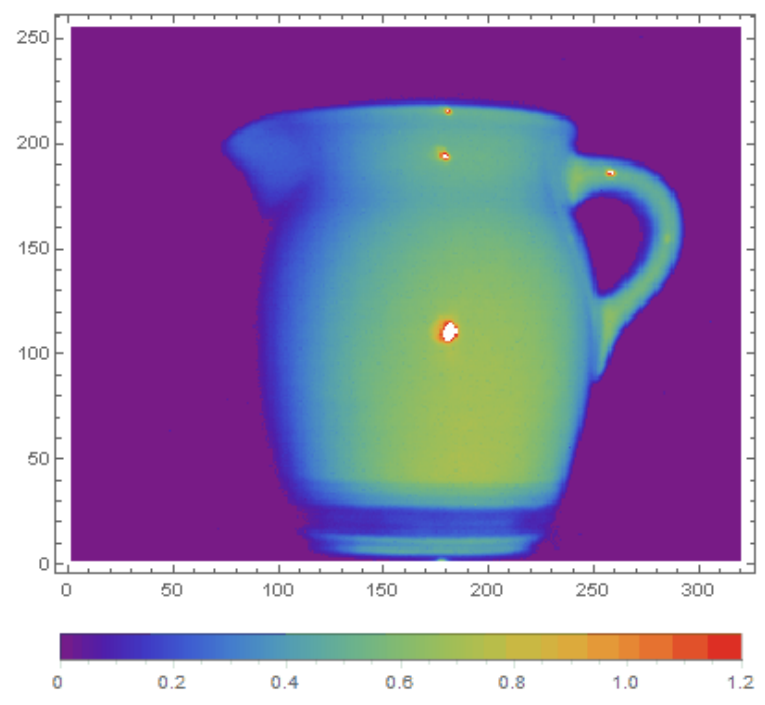

Fig. 5. Reflectance image of a ceramic jug used as a sample. This is a white jug with $11 \mathrm{~cm}$ in height. The sample was illuminated using light with a wavelength of $662 \mathrm{~nm}$ at $30^{\circ}$ from the camera axis. The scale is in arbitrary units.

Acknowledgment. The authors thank Marco Favela from the University of Arizona College of Optical Sciences for his assistance with Solidworks drawings. Juan Manuel López-Téllez acknowledges a postdoctoral research grant from CONACyT, Mexico.

\section{References}

1. R. M. A. Azzam and N. M. Bashara. Ellipsometry and Polarized Light. (Elsevier, 1997).

2. J. M. López-Téllez, N. C. Bruce, and O. G. Rodríguez-Herrera, Appl. Opt. 55, 6025 (2016).

3. F. J. Martínez, A. Márquez, S. Gallego, J. Francés, I. Pascual, and A. Beléndez, Opt. Lett. 39, 1011 (2014).

4. L. B. Wolff, Image Vis. Comput. 15, 81 (1997).

5. J. S. Tyo, D. L. Goldstein, D. B. Chenault, and J. A. Shaw, Appl. Opt. 45, 5453 (2006).

6. O. Morel, C. Stolz, F. Meriaudeau, and P. Gorria, Appl. Opt. 45, 4062 (2006).

7. J. M. Bueno, J. Hunter, C. Cookson, M. Kisilak, and M. Campbell, J. Opt. Soc. Am. A 24, 1337 (2007).

8. M. Karnoukian, S. Faisan, Ch. Heinrich, A. Lallement, and J. Zallat, Opt. Express 23, 18218 (2015).

9. P. Terrier, V. Devlaminck, and J. M. Charbois, J. Opt. Soc. Am. A 25, 423 (2008).

10. M. Kupinski, R. Chipman, Proc. SPIE 9853, 98530P (2016).

11. L. Cartron, N. Josef, A. Lerner, S.D. McCusker, A-S. Darmaillacq, L. Dickel, and N. Shashar, J. Exp. Mar. Biol. Ecol. 447, 80 (2013).

12. R. A. Chipman, W. S. T. Lam, G. Young, Polarized Light and Optical Systems (Optical Sciences and Applications of Light), 1st ed. (CRC Press, 2018).

13. F. Goudail and J. S. Tyo, J. Opt. Soc. Am. A 28, 46 (2011).

14. D. Goldstein, Polarized Light, 2nd ed. (CRC Press, 2003).

15. J. M. López-Téllez, N. C. Bruce, and R. Nava-Sandoval, in Frontiers in Optics 2015, OSA Technical Digest (Optical Society of America, 2015), p. JTu4A.35.

16. S. Alali, A. Gribble, and I. A. Vitkin, Opt. Lett. 41, 1038 (2016).

17. P. Raman, K. Fuller, D. Gregory, M. Newchurch, J. Christy, Proc. SPIE 8160, 816013 (2011).

18. D. Miyazaki, M. Saito, Yoichi Sato, and K. Ikeuchi, J. Opt. Soc. Am. A 19, 687 (2002).

19. L. B. Wolff, IEEE Trans. Pattern Anal. Mach. Intell. 12, 1059 (1990).

20. S. Rahmann, in Proceedings of the International Conference on Pattern Recognition (IEEE Computer Society, 2000), p. 542

21. D. Miyazaki, T. Shigetomi, M. Baba, R. Furukawa, S. Hiura, N. Asada, Optical Engineering 56, 041303 (2016).

22. See http://www.axometrics.com/index.php/products/axostep for more information on the AxoStep Imaging Mueller Matrix Polarimeter.

23. M. H. Smith, Appl. Opt. 41, 2488 (2002).

24. R. A. Chipman, Handbook of Optics, M. Bass (McGraw-Hill, 1995), Chap. 22.

25. M. R. Spiegel, Schaum's Outline of Theory and problems of Statistics, Third Edition, (McGraw-Hill, U.S.A., 1999).

26. S-Y Lu and R.A. Chipman, J. Opt. Soc. Am. A 13, 1106 (1996).

27. J. J. Gil and E. Bernabeu, Opt. Acta 33, 185 (1986).

28. P-Y Gerligand, M. H. Smith, and R. A. Chipman, Opt. Express 4, 420 (1999). 


\section{Full references:}

1. R. M. A. Azzam and N. M. Bashara. Ellipsometry and Polarized Light. (Elsevier, 1997).

2. J. M. López-Téllez, N. C. Bruce, and O. G. Rodríguez-Herrera, "Characterization of optical polarization properties for liquid crystal-based retarders," Appl. Opt. 55(22), 6025-6033 (2016).

3. F. J. Martínez, A. Márquez, S. Gallego, J. Francés, I. Pascual, and A. Beléndez, "Retardance and flicker modeling and characterization of electro-optic linear retarders by averaged Stokes polarimetry," Opt. Lett. 39, 1011-1014 (2014).

4. L. B. Wolff, "Polarization vision: a new sensory approach to image understanding," Image Vis. Comput. 15, 81-93 (1997).

5. J. S. Tyo, D. L. Goldstein, D. B. Chenault, and J. A. Shaw, "Review of passive imaging polarimetry for remote sensing applications," Appl. Opt. 45, 5453-5469 (2006).

6. O. Morel, C. Stolz, F. Meriaudeau, and P. Gorria, "Active lighting applied to three-dimensional reconstruction of specular metallic surfaces by polarization imaging," Appl. Opt. 45, 4062-4068 (2006).

7. J. M. Bueno, J. Hunter, C. Cookson, M. Kisilak, and M. Campbell, "Improved scanning laser fundus imaging using polarimetry," J. Opt. Soc. Am. A 24, 1337-1348 (2007).

8. M. Karnoukian, S. Faisan, Ch. Heinrich, A. Lallement, and J. Zallat, "Segmentation of Mueller matrix images under non-uniform illumination," Opt. Express 23, 18218-18235 (2015).

9. P. Terrier, V. Devlaminck, and J. M. Charbois, "Segmentation of rough surfaces using a polarization imaging system," J. Opt. Soc. Am. A 25, 423430 (2008).

10. M. Kupinski, R. Chipman, "Power spectra trends in imaging polarimetry of outdoor solar illuminated scenes", Proc. SPIE 9853, Polarization: Measurement, Analysis, and Remote Sensing XII, 98530P (4 May 2016).

11. L. Cartron, N. Josef, A. Lerner, S.D. McCusker, A-S. Darmaillacq, L. Dickel, and N. Shashar, "Polarisation vision can improve object detection in turbid waters by cuttlefish," J. Exp. Mar. Biol. Ecol. 447, 80-85 (2013).

12. R. A. Chipman, W. S. T. Lam, G. Young, Polarized Light and Optical Systems (Optical Sciences and Applications of Light), 1st ed. (CRC Press, 2018).

13. F. Goudail and J. S. Tyo, "When is polarimetric imaging preferable to intensity imaging for target detection?," J. Opt. Soc. Am. A 28, 46-53 (2011).

14. D. Goldstein, Polarized Light, 2nd ed. (CRC Press, 2003).

15. J. M. López-Téllez, N. C. Bruce, and R. Nava-Sandoval, "Scanning Polarimetric Scatterometer for Two-Dimensional Rough Surfaces," in Frontiers in Optics 2015, OSA Technical Digest (online) (Optical Society of America, 2015), paper JTu4A.35.

16. S. Alali, A. Gribble, and I. A. Vitkin, "Rapid wide-field Mueller matrix polarimetry imaging based on four photoelastic modulators with no moving parts," Opt. Lett. 41, 1038-1041 (2016)

17. P. Raman, K. Fuller, D. Gregory, M. Newchurch, J. Christy, "Broadband (UV-VIS-NIR) Mueller matrix polarimeter", Proc. SPIE 8160, Polarization Science and Remote Sensing V, 816013 (9 September 2011).

18. D. Miyazaki, M. Saito, Yoichi Sato, and K. Ikeuchi, "Determining surface orientations of transparent objects based on polarization degrees in visible and infrared wavelengths," J. Opt. Soc. Am. A 19, 687-694 (2002).

19. L. B. Wolff, "Polarization-based material classification from specular reflection,” IEEE Trans. Pattern Anal. Mach. Intell. 12, 1059-1071 (1990).

20. S. Rahmann, "Polarization images: a geometric interpretation of shape analysis," in Proceedings of the International Conference on Pattern Recognition (IEEE Computer Society, Los Alamitos, Calif., 2000), pp. 542546.

21. D. Miyazaki, T. Shigetomi, M. Baba, R. Furukawa, S. Hiura, N. Asada, "Surface normal estimation of black specular objects from multiview polarization images," Optical Engineering 56(4), 041303 (30 September 2016).
22. See http://www.axometrics.com/index.php/products/axostep for more information on the AxoStep Imaging Mueller Matrix Polarimeter.

23. M. H. Smith, "Optimization of a dual-rotating-retarder Mueller matrix polarimeter," Appl. Opt. 41(13), 2488-2493 (2002).

24. R. A. Chipman, Handbook of Optics, M. Bass, ed. (McGraw-Hill, 1995), Chap. 22.

25. M. R. Spiegel, Schaum's Outline of Theory and problems of Statistics, Third Edition, (McGraw-Hill, U.S.A., 1999).

26. S-Y Lu and R.A. Chipman, "Interpretation of Mueller matrices based on polar decomposition," J. Opt. Soc. Am. A 13, 1106-1113 (1996).

27. J. J. Gil and E. Bernabeu, "Depolarization and polarization indices of an optical system," Opt. Acta 33, 185-189 (1986).

28. P-Y Gerligand, M. H. Smith, and R. A. Chipman, "Polarimetric images of a cone," Opt. Express 4, 420-430 (1999). 